\title{
Norois
}

Environnement, aménagement, société

\section{Quelle articulation entre identité campagnarde et identité urbaine dans les ménages " périurbains »?}

Which articulation between country identity and urban identity for the households of peri-urban spaces

\section{Rodolphe Dodier}

\section{OpenEdition}

\section{Journals}

Édition électronique

URL : http://journals.openedition.org/norois/1616

DOI : $10.4000 /$ norois. 1616

ISBN : 978-2-7535-1550-5

ISSN : $1760-8546$

\section{Éditeur}

Presses universitaires de Rennes

\section{Édition imprimée}

Date de publication : 1 mars 2007

Pagination : 35-46

ISBN : $978-2-7535-0457-8$

ISSN : 0029-182X

\section{Référence électronique}

Rodolphe Dodier, "Quelle articulation entre identité campagnarde et identité urbaine dans les ménages « périurbains »? », Norois [En ligne], 202 | 2007/1, mis en ligne le 01 mars 2009, consulté le 19 avril 2019. URL : http://journals.openedition.org/norois/1616 ; DOI : 10.4000/norois.1616 


\title{
QUELLE ARTICULATION ENTRE IDENTITÉ CAMPAGNARDE \\ ET IDENTITÉ URBAINE DANS LES MÉNAGES «PÉRIURBAINS »?
}

\author{
RODOLPHE DODIER \\ GREGUM - UMR ESO 6590 CNRS \\ (Université du Maine - Le Mans), \\ avenue Olivier-Messiaen - 72085 LE MANs cedex 9 \\ rodolphe.dodier@univ-lemans.fr
}

\begin{abstract}
RÉSUMÉ
Les espaces périurbains se caractérisent par une forte domination des familles, mais sont de plus en plus hétérogènes sur le plan socio-économique, entre les différentes couronnes et selon les segments du marché immobilier. Les modes de vie périurbains sont donc variés, avec un fort rapport au logement caractéristique des familles, un rapport au village périurbain variant entre fort sentiment identitaire et commune-dortoir, un rapport à la ville oscillant entre attraction et répulsion. L'identité spatiale des ménages périurbains se décline donc en quatre groupes selon leurs rapports à l'urbanité et à la ruralité.
\end{abstract}

MotS CLÉS : Groupes sociaux - Habiter - Identité spatiale - Périurbain.

\section{ABSTRACT \\ Which articulation between country identity and urban identity for the households of peri-urban spaces}

Peri-urban spaces are characterized by the predominance of families but are more and more heterogeneous on a socio-economic basis. Differences between suburban zones and between different types of housing are great. Peri-urban ways of life are also very varied. Families have a strong relationship with their home. As far as their relationship with the peri-urban village is concerned fluctuation belween strong identification with village life of indifference to what is experienced as a dormitory town is noticed. Relationship with the town oscillates between attraction and repulsion. Four types of spatial identity of periurban households have been identified according to their relationship with both urban and country life.

KEY WORDS : Dwelling - Peri-urban Space - Social Groups - Spatial Identity.

Résider à la campagne, travailler et consommer en ville, avoir des loisirs et des relations sociales dans les deux espaces, cette division spatiale de l'espace vécu est devenue le lot quotidien de nombreux ménages périurbains. Comment ces ménages articulent-ils les deux espaces de vie, qu'est-ce qui «fait campagne » dans leur mode de vie, quelles sont les conséquences d'un espace de vie éclaté sur leur identité spatiale? 
Répondre à ces questions implique de partir d'une comparaison entre différents types d'espaces. Les discours sur le périurbain et notamment la thèse assez courante d'une sociabilité de l'entre-soi dominante dans ce type d'espace, se base trop souvent sur une analyse sans base comparative ou avec comme seule base de comparaison le propre vécu de chercheurs habitant dans l'espace central des villes et ayant une lecture positive des interactions sociales en ville (justifiant leur propre pratique de l'espace et des relations sociales). Analyser la situation dans le périurbain nécessite donc d'utiliser une approche comparative, entre l'espace urbain, l'espace périurbain et l'espace rural, seule méthode garantissant de ne pas biaiser l'analyse par le propre vécu du chercheur. Une comparaison entre le périurbain proche des villes et le périurbain lointain permet également de sortir des discours trop généraux et de distinguer les différentes situations dans l'espace périurbain, depuis les espaces proches des villes où les professions intermédiaires dominent, parfois en association avec les cadres dans les plus grandes métropoles, jusqu'aux communes les plus éloignées de la ville, refuge des catégories populaires solvables même si certains ménages sont touchés par le surendettement. Dans les campagnes vivantes de la France de l'Ouest, il existe un espace périurbain bien identifiable autour des villes de toutes tailles, non seulement autour des métropoles régionales, des villes intermédiaires et des villes moyennes, mais aussi autour de plus petites villes. Il existe également un large espace rural non polarisé, au sens INSEE du terme (par référence aux aires urbaines), même si la distance à l'urbain n'est jamais très élevée, avec un continuum de situations intermédiaires qui rendent la région particulièrement intéressante à étudier, permettant notamment de mieux isoler le rôle de la taille urbaine dans les différents processus.

Quels sont les ménages qui résident dans ces différents types d'espace? Une part non négligeable des ménages restent, quoi qu'en disent certains, profondément ruraux dans leurs modes de vie et de pensée, même s'ils fréquentent les villes pour la consommation ou des formes de loisirs, même s'ils ont adopté des manières d'habiter généralement proches de leurs voisins périurbains. Ils coexistent avec des ménages qui résident dans l'espace rural, qui y projettent leurs valeurs et leurs représentations, mais qui travaillent, consomment, ont de nombreuses relations sociales dans la ville plus ou moins éloignée. Là encore, il existe un continuum de situations sociales, des modes de vie les plus ruraux aux modes de vie totalement urbains. Montrer la part relative de ces différents types de ménages et son évolution récente (existe-t-il réellement un mouvement de gentrification rurale ?), permettra de brosser un portrait fidèle de la diversité du périurbain.

Quelles sont les conséquences pour la dynamique sociale de ces espaces? S'oriente-t-on vers des campagnes qui ne seraient qu'une figure de l'urbain, vers une résistance acharnée des valeurs rurales, ou vers l'émergence d'une réelle nouvelle figure sociale : le ménage périurbain? Un ensemble d'entretiens menés pour apprécier l'habiter des différents types de population (35 entretiens qualitatifs), sur les lieux fréquentés, les pratiques spatiales, les valeurs et représentations associées à l'espace, permet de proposer quelques pistes de réponse. La nature du parcours antérieur, la façon dont les individus s'investissent ou non dans la vie locale, les origines sociales, l'appréciation de la mobilité, etc., sont certains des facteurs de différenciation sociale que l'on peut mettre en avant. Cela permet aussi de ne pas raisonner seulement selon une grille de lecture binaire qui opposerait les valeurs rurales et les valeurs urbaines, mais de proposer plutôt une lecture circulaire entre valeurs rurales et urbaines, des positionnements typiquement urbains pouvant rejoindre, dans leurs manifestations, des manières d'être caractéristiques du rural. Au final, il semble possible de montrer quelles sont les différentes articulations possibles entre identité urbaine et identité rurale pour ces populations.

\section{La diversité socio-spatiale du périurbain}

La composition sociale du périurbain n'est pas toujours bien connue et bien identifiée. Des travaux à l'échelle de la France, avec un certain nombre de zooms sur certaines grandes villes (Guilluy et Noyé, 2004) ont pourtant permis de globalement bien caractériser les populations habitant dans le périurbain et les dynamiques en cours. Les employés et les ouvriers sont de plus 
en plus surreprésentés au fur et à mesure qu'on s'éloigne de la ville, alors que le mouvement est inverse pour les professions intermédiaires et a fortiori pour les cadres supérieurs, toujours peu présents dans ce type d'espace. Les mouvements en cours tendent à renforcer cette opposition entre centres des grandes villes et leur espace périurbain lointain, générant une «nouvelle fracture » à la fois sociale et spatiale, qui s'ajoute à la polarisation sociale à l'œuvre dans les grandes villes. Toutefois, dans les travaux moins descriptifs, l'expression de cette composition sociale est parfois un peu réductrice, oscillant entre « espace des catégories moyennes » et « la place du peuple » (Estèbe, 2004) et surtout insistant sur l'apparente homogénéité des habitants du périurbain, cause supposée de l'entre soi.

La diversité sociale est certes sans doute un peu plus faible que dans l'espace urbain, avec en particulier moins de catégories sociales aisées et moins de catégories paupérisées mais derrière les effets de lissage engendrés par une lecture exclusivement en termes de moyenne, tous les groupes sociaux sont bien représentés. Encore faudrait-il pondérer le constat de la moindre diversité par une analyse selon différentes échelles, les quartiers des grandes villes, du moins ceux composés par des catégories extrêmes, étant eux aussi de plus en plus homogènes. Plus encore, il faudrait analyser les réelles relations sociales inter-catégories, la diversité statistique n'étant pas forcément synonyme de mixité dans les relations sociales.

En fait, on retrouve au niveau du périurbain les mêmes variations que dans les quartiers des grandes villes, zones sensibles et beaux quartiers exceptés, avec également des modes de vie fortement variés, des plus ruraux aux plus urbains. Cela est dû d'une part à la diversité même des situations que l'on peut regrouper sous le vocable de périurbain, d'autre part à la diversité, dans une couronne donnée, des populations résidentes.

Dans les aires urbaines, les processus aboutissant à la composition sociale de l'espace périurbain sont désormais bien connus. Sous l'influence du fonctionnement du marché immobilier, les catégories populaires solvables se retrouvent massivement dans le périurbain, rejetées de plus en plus loin en périphérie par l'augmentation importante, notamment depuis 1988, des coûts fonciers. L'accession à la propriété n'est possible, pour ces catégories, qu'au prix de dépenses de transport importantes, avec un fort éloignement des infrastructures scolaires, au prix également d'un manque cruel d'équipements collectifs, notamment en matière de garde d'enfants et d'animation culturelle et sportive (sauf le foot!), ce qui génère des sentiments de relégation. Cette perception de l'éloignement, à la fois physique et politique, est confortée par les difficultés d'un certain nombre de ménages face au remboursement des prêts immobiliers, en particulier en raison de la précarité des situations socioprofessionnelles avec de fortes variations des revenus dans le temps, et des contraintes imposées par les distances à parcourir, qui incitent parfois les femmes à cesser de travailler ce qui rend la situation financière du ménage difficile (Rougé, 2005). Cet ensemble de facteurs peut effectivement provoquer des formes de repli sur soi et éventuellement des comportements politiques spécifiques (fort vote Front National), encore que la composition sociale forme de ce point de vue un effet de structure souvent déterminant (surreprésentation des ouvriers dans le vote Front National).

L'espace rural au sens large (incluant les petites villes qui l'animent) et peut-être plus encore l'espace périurbain entourant ces petites villes, sont désormais les véritables espaces d'accueil des employés et surtout des ouvriers. Dans les campagnes vivantes de l'Ouest de la France, même mis à mal par la concurrence des pays à bas salaires, le système fordiste des petites villes industrielles reste bien vivant, avec ici ou là de grosses difficultés (Mamers avec le départ de Moulinex), mais aussi de belles réussites avec un développement économique continu depuis plus de 30 ans. Cet espace rural, non polarisé par les aires urbaines, est également marqué par un processus de périurbanisation alors que les facteurs explicatifs habituellement utilisés pour l'espace urbain n'y ont pas la même vigueur. Les différences de coûts fonciers et immobiliers ne sont pas très fortes, l'offre en matière de logement dans les petites villes n'est pas complètement saturée et les aménités environnementales ne sont pas beaucoup plus faibles que dans l'espace rural environnant, où les pollutions d'origine agricole, notamment au niveau des odeurs, contrebalancent bien les pollutions 
d'origine industrielle des petites villes. Ce mouvement de périurbanisation est en particulier soutenu par la population ouvrière qui afflue massivement dans ce type d'espace, pour retrouver un cadre de vie rural, pour devenir propriétaire, pour se rapprocher de ses racines. Les explications d'ordre identitaire sont en effet les premières explications de ce mouvement.

Toutefois, la vraie homogénéité de l'espace périurbain, qu’il soit situé autour des grandes aires urbaines ou des petites villes, se situe au niveau des variables socio-démographiques. Autant la ville-centre est le lieu d'accueil des types de ménages emblématiques de la société contemporaine (ménages d'une seule personne, ménages monoparentaux, ménages sans famille), autant la composition du périurbain est marquée par les familles avec enfants. Quels que soient les segments de l'habitat qui sont occupés, les familles d'un à trois enfants sont surreprésentées, même si parfois les enfants devenus grands commencent à partir (et à retourner en ville pour former les ménages décrits précédemment...). Selon la date de construction du lotissement, selon des cycles générationnels dans les autres types d'habitat, on observe un renouvellement de la population. Il s'explique par l'arrivée massive de ménages ayant de jeunes enfants (ou ayant l'intention d'en avoir) et le départ de ménages plus âgés, au moment de la retraite, mais également avec de grands adolescents, souvent en raison des difficultés de transports de cette catégorie d'âge.

\section{Composition sociale selon les types d'habitat}

La composition sociale de ces espaces périurbains est donc marquée par les familles des catégories moyennes et populaires (mais globalement solvables), dans un contexte de mixité sociale non négligeable. L'exemple du périurbain situé au nord du Mans permet de repérer la présence de différentes catégories sociales dans plusieurs segments du marché immobilier, ce qui rejoint assez bien le fonctionnement constaté dans les grandes villes.

La forme spatiale dominante dans le paysage est bien sûr le lotissement, souvent décrié pour la monotonie du bâti et l'absence de recherche architecturale. Dans ces lotissements, la population est d'une mixité très variable et dépend étroitement de la diversité de la taille des parcelles. Toutefois, à bâti identique, on peut noter que la composition sociale des parcelles hors lotissement n'est pas foncièrement différente, en particulier lorsqu'elles forment des bandes continues aux entrées et sorties de village. Dans les premières couronnes périurbaines des grandes villes, les situations sociales sont globalement plus spécifiques des catégories moyennes, avec cependant des variations importantes selon le type d'habitat. Ces catégories sont plus particulièrement présentes dans les lotissements qui font la spécificité du paysage des premières couronnes périurbaines, encore faut-il nuancer selon l'ancienneté de la construction (plus le lotissement est ancien plus la proportion d'ouvriers augmente puisque le coût relatif du foncier était alors plus faible), selon la taille des parcelles (avec une stratégie parfois explicite de la part de certaines communes de la première couronne de faire du tri social par ce biais) et selon la situation par rapport aux axes de circulation ou la proximité des équipements, en particulier scolaires (assez près pour l'utilisation, mais pas trop à cause des désagréments environnementaux, en particulier le bruit généré par les axes de transport). Plus en périphérie, les situations sociales sont plus modestes, le coût au $\mathrm{m}^{2}$ diminuant régulièrement avec la distance, mais la taille des parcelles tend également à diminuer légèrement, renforçant l'homogénéité sociale. Il s'agit cependant d'une homogénéité sociale par défaut, par absence relative des autres catégories sociales, et non d'une volonté délibérée de vivre entre soi. Ces lotissements éloignés de la ville-centre (souvent plus de $30 \mathrm{~km}$ ) ont en fait quasiment la même composition sociale que le périurbain situé autour des petites villes, où la forte proportion d'ouvriers est l'indicateur le plus spécifique. Là encore, l'homogénéité sociale est plus une conséquence du fonctionnement du marché immobilier qu'une volonté explicite de certaines catégories sociales de vivre entre soi.

Dans le périurbain, il existe peu de situations sociales vraiment difficiles, sauf dans le logement très social qui commence à être implanté dans certaines communes, et qui génère effectivement parfois des phénomènes de rejet, en particulier lorsque l'accueil de familles cumulant difficultés 
professionnelles et familiales met à mal la relative tranquillité de la commune. Les problèmes se situent plutôt dans le registre de l'incivilité, plus rarement de la petite délinquance, mais une frange de la population, surtout la plus populaire, est justement arrivée dans le périurbain pour éviter l'ambiance sociale des ZUP, parfois jamais fréquentées pourtant. Il existe également une couche spécifique de population très présente dans les vieilles maisons de bourg (segment vraiment peu connu dans la littérature scientifique). Ce type d'habitat se caractérise par des maisons de petite taille, souvent assez peu confortables, (c'est le segment prioritairement visé dans les politiques d'amélioration de l'habitat), dénuées des aménités environnementales habituellement présentes dans le périurbain (pas ou peu de terrain, maisons mitoyennes, etc.). Une population différente est présente dans ce segment, avec des ménages beaucoup plus âgés et d'origine rurale, avec une proportion non négligeable de situations de pauvreté, ou alors des ménages plus jeunes et sans enfants, avec assez souvent des problèmes d'insertion professionnelle (forte précarité du travail, problèmes financiers liés aux coûts de transport, etc.). Dans ce dernier cas, les ménages, parfois des personnes seules, n’ont pas trouvé de résidence dans le segment locatif privé de la ville proche, complètement saturé ou à des coûts prohibitifs, et ne désirent pas (ou n'ont pas pu dans certains cas) intégrer le segment du locatif social.

Enfin, les catégories aisées voire très aisées, ne sont pas absentes non plus de l'espace périurbain, mais elles se concentrent essentiellement dans les maisons isolées, pavillons contemporains ou fermettes restaurées. Il s'agit d'un segment de l'immobilier bien spécifique, avec même ses acteurs spécialisés (certaines agences immobilières, les notaires), dans lequel les coûts fonciers et immobiliers augmentent actuellement de façon forte et régulière autour des grandes aires urbaines (forte proportion de cadres supérieurs), même si la grande majorité des cadres continuent de vouloir résider dans l'urbain dense. Cette population coexiste dans ce qui reste de l'espace agricole dans le périurbain avec d'autres populations bien différentes. Le premier groupe est formé par les «transformeurs-bricoleurs ", de condition sociale souvent plus modeste mais globalement assez variée. Ils occupent le même type de bâti mais en assurent eux-mêmes la réhabilitation (alors que les cadres utilisent les services des artisans), parfois avec l'aide d'un réseau d'amis spécialisés. Certains en sont à leur deuxième ou troisième maison à restaurer, ils y passent tout leur temps libre et leurs vacances, mais dégagent parfois des plus-values non négligeables. Le second groupe est constitué des agriculteurs ou anciens agriculteurs, au mode de vie un peu différent, ce qui génère parfois quelques conflits larvés.

Dans le périurbain des petites villes de l'espace rural, la population agricole est plus importante mais les catégories aisées sont beaucoup moins présentes, car elles sont moins nombreuses dans les petits bassins d'emploi (structure d'emploi souvent industrielle et peu qualifiée) et résident plus généralement dans les maisons bourgeoises des bourgs-centres. Dans ces espaces, les fermettes rénovées sont plutôt la propriété de résidents secondaires, parfois d'origine francilienne ou étrangère, dont quelques-uns tendent à résider de façon pérenne depuis quelques années. Le mouvement est cependant encore faible, sans commune mesure avec des espaces avec de très fortes aménités environnementales ou culturelles (Périgord, Alpes du Sud, etc.), ce qui ne semble pas permettre de parler de gentrification rurale (Raymond, 2003). Cette présence des résidents secondaires, qui investissent parfois beaucoup, à la fois au sens premier par la rénovation, mais aussi au sens identitaire, assure un minimum de mixité sociale, la fourchette des situations sociales allant finalement de la misère rurale à la bourgeoisie aisée, ce qui ne veut pas dire non plus que toutes ces catégories sociales communiquent facilement.

En fait, la composition sociale ne correspond que très imparfaitement aux différents modes de vie. Certains ménages qu'on pourrait classer comme catégories moyennes urbaines peuvent avoir des relations à l'espace très proche de ménages ruraux, inversement d'autres restent très profondément attachés à la ville, non seulement par leurs pratiques spatiales (travail, consommation, etc.), mais aussi par leur identité spatiale. 


\section{Modes de vie périurbains}

Un certain nombre d'indices permettent en fait de caractériser la façon d'habiter le périurbain, et qui ne correspondent pas exactement aux différenciations sociales ni même aux pratiques spatiales (travailler en ville ou non). Marquer sa différence ou son appartenance, explicitement ou implicitement, va passer par la mise en scène de l'habitat et l'utilisation du logement, par la nature de l'utilisation du terrain, par le rapport au village périurbain et par le rapport à la ville, permettant ainsi de caractériser l'identité du ménage entre rural et urbain.

\section{LE RAPPORT AU LOGEMENT}

Comme les familles sont particulièrement présentes dans le périurbain, la constante la plus évidente concerne le rapport au logement. Dans la plupart des ménages, ce rapport est assez fort, plus semble-t-il que dans le centre des grandes villes (Authier, 2001) et est partagé par la population rurale plus anciennement installée. Le désir de devenir propriétaire, très présent dans cette population, les difficultés parfois pour trouver le financement ou pour conserver son statut, génèrent un attachement important au logement et le fait de devoir partir est vécu comme un déchirement, que ce soit pour des raisons de surendettement, de difficultés professionnelles ou de difficultés familiales. Le fait d'avoir également passé du temps à la finition des travaux (peintures et papiers peints) ou d'avoir transformé son logement (aménagement des combles, modification de l'agencement intérieur, etc.) renforce ce sentiment, également conforté par le temps important passé dans le logement, caractéristique des familles.

Des différenciations existent cependant dans l'utilisation du logement, en particulier dans les pièces les plus pratiquées. La grande pièce à vivre, caractéristique des pavillons contemporains ou de certaines fermettes rénovées, est un élément de classification sociale car essentiellement présent dans les logements de ménages aisés. Mais l'ameublement peut être assez varié, du style néo-rural (le mot rustique est souvent employé bien que le confort ne soit pas sacrifié) qui n’est pas forcément le fait de vrais ruraux bien au contraire, jusqu'au mobilier le plus contemporain. Les options les plus spécifiques sont des marqueurs sociaux alors que les ménages populaires sont globalement beaucoup plus classiques dans leurs options de décoration. La cuisine reste par contre une pièce essentielle pour les ménages populaires, et ne permet d'ailleurs pas toujours de distinguer ménages d'origine rurale (agriculteurs) et d'origine urbaine (ouvriers), notamment autour des petites villes, sans doute parce que ces derniers ont souvent, dans notre espace d'analyse, une origine rurale pas si ancienne que cela. L'importance accordée à l'atelier (notamment pour les «transformeurs ») est également facteur de renforcement de l'attachement au logement.

L'extérieur du logement est sans doute plus un marqueur de l'appartenance urbaine ou rurale qu'un marqueur de classification sociale. La présence d'une pelouse, de nombreuses fleurs, d'arbres d'ornement, ou au contraire d'un potager et d'arbres fruitiers, distingue en partie les modes de vie plus urbains ou plus ruraux. Toutefois, la lecture n'est pas toujours évidente, les tendances les plus récentes en matière d'ornement ramenant le potager en bonne place, avec même un attrait pour les légumes rares de plus en plus fréquent. Mais il subsiste des différences entre le potager utilitaire, qu'on peut considérer comme émanant de valeurs rurales, et d'autres formes plus composites, en particulier à partir de la présence de l'herbe à côté ou dans le potager. Dans le milieu rural, l'herbe est historiquement nourricière et non un élément de décoration ou de confort. Au contraire, certaines personnes d'origine rurale considèrent encore que la présence d'herbe à proximité de l'habitation est la marque d'un mauvais entretien du terrain. Les abords immédiats de la maison, les allées du potager et ses alentours restent donc exempts d'herbe, même si on sacrifie un peu à la mode urbaine en ayant un carré de pelouse (mais bien net!) dans un coin du jardin. A contrario, les jardins "sauvages », ou mêlant étroitement légumes et fleurs, ou tout simplement sans potager, sont vraiment des indicateurs assez fiables de modes de vie urbains, confirmés par les pratiques spatiales. 


\section{LE RAPPORT AU VILLAGE PÉRIURBAIN}

Le rapport au village périurbain est également assez disparate et dépend aussi de la distance à la ville-centre. Un certain nombre de ménages expriment, en sus de raisons financières ou de taille de logement, des raisons liées à la sociabilité du village comme ayant participé à la décision de s'installer dans le périurbain. Parfois, il s'agit même d'un « retour aux sources », un aïeul ou un membre de la famille ayant pu résider dans la commune finalement choisie, ou du moins dans une commune relativement proche. Il existait donc une certaine familiarité avec l'espace finalement choisi pour l'installation dans le périurbain, même si d'autres raisons sont en fait plus importantes, tenant compte à la fois des caractéristiques du logement et d'un ensemble de proximités relatives (Dodier, 2004a). Fuir l'anonymat de la grande ville, retrouver de «vraies relations avec les gens », autant de manières d'exprimer l'attirance pour une sociabilité villageoise idéalisée. Il s'agit sans doute d'un discours largement reconstruit, très dans l'air du temps, exprimé à partir de la nostalgie de valeurs considérées comme rurales ou émanant de la «campagne ». Dans la réalité, les déceptions sont aussi nombreuses, soit en raison du fort contrôle social qui fait que tout se sait dans la commune (et qu'être un peu différent dans sa manière de vivre peut être mal vécu en raison du comportement des voisins), soit en raison du décalage entre les attentes du ménage et la réalité vécue (voisins méfiants, notamment dans la partie agricole de la commune périurbaine). Dans les lotissements, des sortes de cycles peuvent être observés, avec une ambiance souvent décrite comme idyllique lors des premières années : on se reçoit, on pratique un certain nombre d'activités ensemble, etc. Ensuite (ou parallèlement), les inimitiés se font jour, les problèmes de voisinage prennent des proportions importantes à partir de simples détails (les haies mitoyennes, le bruit dans les parties extérieures, les poubelles, etc.), les relations sociales se détériorent, parfois trop de proximité finit par générer des problèmes de couple (qu'on se plaît à relater lorsqu'il s'agit de voisins peu appréciés), bref la réalité des relations sociales prend des formes variées, de l'entente cordiale à la franche hostilité.

En fait, le rapport au village périurbain semble aussi varié que le rapport au quartier en milieu urbain. Certaines personnes sont très impliquées dans la vie sociale locale par le biais des associations ou de la participation à la vie municipale, avec l'archétype du « surinvesti », membre de plusieurs associations, participant à toutes les animations locales. La plupart des ménages ont un rapport plus mesuré, avec des pratiques impliquantes (recevoir chez soi, etc.), mais aussi beaucoup de consommation (de loisirs sportifs ou culturels par exemple) qui les amènent à des relations sociales sans que ce soit forcément un objectif initial. Un ménage présent dans une commune depuis environ cinq ans raconte comment, malgré la méfiance initiale liée à une expérience malheureuse antérieure, ils ont fini par s'insérer fortement dans la vie locale, d'abord par l'école (accompagnement des sorties scolaires puis participation au comité de parents débouchant sur l'organisation de journées festives), puis par les activités associatives. Inversement, d'autres personnes n'ont qu'une pratique limitée de la commune où ils résident, avec une utilisation éventuelle des quelques services et commerces locaux, mais sans véritable implication. Dans un certain nombre de cas, la commune n'est vraiment qu'un lieu de résidence sans aucun écho identitaire, sans aucune véritable pratique spatiale ou sociale.

Le lien entre types de ménage et haut niveau de pratique spatiale du village périurbain est délicat à trancher sur de faibles effectifs, mais quelques tendances peuvent être proposées. Si les ménages qu'on peut désigner comme « ruraux » sont bien présents dans la vie de la commune, leur pratique est centrée sur un petit nombre d'activités, souvent assez caractéristiques (chasse, anciens d'AFN, clubs des aînés ruraux, comice agricole, etc.), même s'ils ne sont pas les seuls à fréquenter les associations qui portent ces activités. Ils utilisent aussi assez couramment le commerce local, notamment le café (encore que lorsqu'il y a plusieurs cafés, ceux-ci soient souvent spécialisés sur un type de population, jeune ou rurale, et s'il n'y en qu'un, il peut exister une spécialisation selon les heures - le matin - ou les salles ou la place dans la salle - le comptoir -). Les ménages aisés ont également une forte pratique de l'espace du village périurbain, par volonté délibérée de soutenir 
le commerce local ou en recherchant les «vrais produits du terroir », ou bien en étant souvent à l'origine des associations culturelles (musique, voire des activités plus rares comme le théâtre) ou de celles organisant les activités destinées aux enfants (écoles, activités sportives éventuellement). La pratique du village périurbain est par contre plus faible pour les catégories moyennes et plus encore pour les catégories populaires, même s'il existe évidemment des exceptions remarquables. Ces catégories révèlent parfois des attitudes de repli sur le logement, mais c'est également le cas en milieu urbain.

\section{LE RAPPORT À LA VILLE}

Le rapport à la ville voisine est également un élément qui peut permettre de distinguer les modes de vie urbains et ruraux. D'une part, parce que certains y travaillent et font le trajet tous les jours, alors que pour les autres, il faut une volonté délibérée, un besoin spécifique, pour que la ville, et plus spécialement son centre-ville, soit parcourue, fréquentée et éventuellement appropriée. Dans l'espace périurbain, le rapport à la ville oscille toujours entre attraction et répulsion, selon des combinaisons propres à chaque ménage, voire à chaque individu.

La ville reste le lieu de travail dominant, concernant une proportion d'actifs pouvant atteindre $85 \%$ dans des communes à la fois proches des grandes villes et dépourvues du moindre tissu industriel, où les seuls emplois locaux sont les emplois de services à la population (l'école, la mairie, les assistantes maternelles, éventuellement quelques artisans et commerçants, quelques services aux personnes). Dans la première couronne périurbaine des grandes villes, le chiffre est souvent un peu plus faible suite au desserrement d'un certain nombre d'activités industrielles ou commerciales. Ensuite, avec l'éloignement à la ville, la proportion d'actifs travaillant dans le centre du bassin d'emploi diminue alors que celle des actifs travaillant sur la commune de résidence augmente, avec un tissu agricole plus ou moins résiduel, mais aussi d'autres activités, notamment industrielles dans les petites villes. La composition sociale des flux change également, avec une proportion croissante d'ouvriers vers l'extérieur du bassin d'emploi. Le double effet de la distance et du statut social se combine en fait pour expliquer des relations avec la ville de plus en plus ténues avec l'éloignement au centre-ville, bien que la diversité des pratiques soit la règle au sein d'une même couronne.

Dans les premières couronnes, les rapports à l'urbain sont toujours très forts. La consommation a lieu essentiellement en ville, notamment dans les hypermarchés périphériques, mais de nombreuses personnes fréquentent encore le centre-ville dans ce but, notamment pour des achats exceptionnels. Les activités culturelles, pourtant bien développées dans les communes des premières couronnes, ont également parfois lieu en ville, notamment celles des parents (pour les enfants, on a recours aux activités « locales », de la commune ou d'une ou deux communes voisines), et également pour des activités plus rares, absentes dans l'espace périurbain. Cet effet d'ouverture du choix permis par la proximité de la ville joue aussi pour les activités sportives, encore une fois plus particulièrement pour les activités rares. Enfin, pour un certain nombre de ménages périurbains, restés profondément urbains dans leurs modes de vie, les relations de sociabilité les plus importantes restent celles s'exerçant à l'échelle de l'ensemble de la ville proche. On reçoit ou on se déplace chez d'autres ménages répartis sur un vaste ensemble urbain, à partir de relations sociales construites avant l'emménagement dans le périurbain (les amis d'enfance, les collègues de travail, éventuellement les anciens voisins), mais aussi pour des relations sociales construites après l'emménagement, par exemple dans le cadre d'une activité associative. Dans quelques cas, le lieu de résidence périurbain est même devenu le lieu de ralliement du groupe d'amis, avec une place importante faite au barbecue et à l'environnement « campagnard » (qui permet aussi de faire plus de bruit!).

Plus on s'éloigne de la ville, plus distance spatiale et distance sociale se combinent pour expliquer un moindre rapport à l'urbain. Outre la plus faible proportion d'actifs travaillant en ville, on constate également une plus faible proportion de jeunes étudiant en ville (le statut social étant plus faible il y a moins de poursuites d'études dans le supérieur), moins de personnes ayant des activités culturelles en ville (l'offre locale, pourtant plus faible que dans les premières couronnes, 
excepté dans les petites villes, devenant suffisante). La fréquentation des zones commerciales périphériques fait cependant exception, puisque toutes les catégories sociales quels que soient les modes de vie (urbains ou ruraux), fréquentent l'hypermarché ou le supermarché, ainsi que les surfaces spécialisées (même si pour les « ruraux », c’est essentiellement le magasin de bricolage). La seule exception concerne les personnes à faible mobilité, personnes très âgées en particulier, mais aussi jeunes non motorisés, ce qui est souvent source, pour ces derniers, de conflits dans la famille ou dans la commune. Cette grande prudence vis-à-vis de la ville dans le périurbain lointain ne doit pas être interprétée comme un rejet mais plutôt comme une indifférence. Un grand nombre de ces ménages populaires avec de faibles rapports à la ville ont-ils d'ailleurs jamais été urbains? Lors de leur passage dans l'espace urbain, parfois sur deux générations, la ville n’a pas forcément été totalement investie. Ils ont été contraints par les difficultés de transport de l'époque de venir en ville pour travailler mais ont toujours gardé une part d'identité rurale et ne sont donc pas mécontents de retrouver un espace « campagnard », au moins dans sa morphologie.

Entre les deux extrêmes, de nombreux ménages ont une pratique modérée de la ville. Ils la fréquentent ou non pour leur travail, sans que ce soit forcément l'indicateur le plus pertinent de l'attachement à l'urbain; ils jonglent entre l'offre locale et l'offre urbaine, que ce soit pour le commerce ou les activités de loisir; ils ont des relations sociales dans les deux types d'espace sans forcément faire une distinction nette d'ailleurs, avec une sociabilité plutôt tournée vers la famille si elle est présente dans le même département... Ils reconnaissent à la fois l'utilisation des avantages de la ville et un certain attachement à celle-ci (lors de l'identification loin de leur lieu de résidence c'est ainsi qu'ils se présentent) mais revendiquent aussi, sur un mode doux, l'appartenance de leur lieu de résidence à la « campagne ». Concrètement, ils évitent généralement le centre-ville, peu accessible en voiture alors que celle-ci est un élément incontournable de leur mobilité, et fréquentent donc beaucoup plus les pôles périphériques emblématiques de la ville polycentrique. Autour des petites villes, le centre reste bien le lieu urbain emblématique et pratiqué, les difficultés de stationnement étant moins importantes et le développement périphérique encore très limité sauf pour le commerce.

\section{Valeurs et représentations de l'espace}

Le bilan des rapports aux trois enveloppes (Moles, 1992) successives que sont le logement, le village périurbain et la ville dans sa globalité montre donc l'importante hétérogénéité de l'identité spatiale des habitants du périurbain. L'identité spatiale peut être définie par l'ensemble des référents territoriaux ou spatiaux mobilisés de façon active par les individus ou les ménages, aux côtés d'autres référents identitaires tels ceux issus des milieux socioprofessionnels (métier, classe sociale), ethniques, culturels ou familiaux fréquentés. Cette identité spatiale est in fine aussi variée qu'en milieu urbain, où les positionnements de repli sur le logement côtoient une pratique intense et impliquante de l'ensemble de la ville et de ses composantes (Authier, 2001).

Pour apprécier l'identité spatiale des ménages du périurbain, plutôt que de définir une grille préalable de valeurs rurales (ou « campagnardes ») et de valeurs urbaines, il a semblé plus judicieux de partir de la représentation qu'ont les ménages de leur propre identité, même si c'est souvent assez délicat de lire cette identité spatiale derrière les discours construits à partir de référents territoriaux. Les termes de rural et d'urbain sont parfois utilisés, généralement peu dans un sens identitaire, mais plus comme une référence parfois implicite à un certain nombre de valeurs. Le terme de campagne est un peu plus utilisé, essentiellement pour décrire un type d'habitat spécifique et son articulation au fait de travailler en ville, surtout quand il s'agit du centre-ville, et peut donc prendre un sens spécifique d'appartenance à un espace particulier. À partir de 35 entretiens semi-directifs, avec des questions ouvertes sur la façon de se présenter lors de déplacements, sur les représentations et sur l'appréciation de la mobilité, une distinction selon l'ancienneté d'arrivée dans l'espace périurbain paraît plus pertinente qu'une typologie selon le statut social ou même selon la distance au centre. Quatre principaux types de réponses ressortent, avec toutes les limites liées au faible effectif. 
- Le premier groupe facilement identifiable est formé des personnes originaires de la commune ou de communes proches, ayant toujours résidé dans l'espace rural sans passer par la ville. Loin de leur lieu de résidence, ils se présentent plutôt en référence au département de résidence, affinant la réponse par la mention de la commune, même petite, si leur interlocuteur semble connaître la région. La référence à la ville (Le Mans par exemple) est secondaire, ce qui marque très nettement l'appartenance rurale, même dans un espace périurbain de grande ville. L'identité spatiale est réduite pour l'essentiel à la commune de résidence, éventuellement élargie à quelques communes contiguës, souvent plus sur une base concentrique qu'à partir des constructions intercommunales récentes. Le rapport au logement est fort, la pratique de la commune est souvent intense, variant cependant avec l'âge (une grande partie est assez âgée et s'implique moins tout en se sentant très concernée par la vie de la commune). La mobilité vers la grande ville proche est pourtant courante, au moins à l'occasion des achats en hypermarché, mais c'est généralement perçu comme une sortie vers un espace un peu étranger, qui ne fait pas partie de l'espace vécu ordinaire. Ce type d'identité spatiale se retrouve aussi dans le périurbain des petites villes de l'espace rural, avec des relations plus importantes avec la petite ville proche, sans doute parce que les différenciations culturelles sont moins fortes et les distances parcourues moins importantes.

- Le second groupe est plutôt formé par les périurbains de longue date, installés depuis longtemps dans la commune et qui font souvent des réponses alambiquées pour décrire leur appartenance territoriale. Ils insistent beaucoup sur leur commune de résidence comme élément fort de leur identité, en tout cas sur leur présence dans un paysage "campagnard », parfois revendiqué comme un art de vivre, alors même qu'ils travaillent et consomment essentiellement en ville. Le lieu de travail urbain ne génère pas forcément un sentiment d'appartenance très fort, surtout dans les catégories populaires, mais la référence à la ville n'est pourtant jamais loin, soit dans l'évocation de souvenirs anciens et notamment de leur parcours résidentiel, soit dans la façon de se présenter lorsqu'ils sont éloignés de leur domicile (la ville est citée au même niveau que le département). Ils oscillent manifestement entre valeurs rurales et valeurs urbaines, essayant de prendre le meilleur des deux (la qualité de vie dans un espace " préservé ", la vie culturelle de l'espace urbain, qui est aussi le lieu du travail et cela a son importance) sans forcément réussir à échapper à ce qu'ils considèrent comme des défauts dans les deux cas (l'anonymat de la grande ville, l'atonie des relations et le contrôle social en milieu rural). Leur appréciation de la mobilité quotidienne est à l'image de leur rapport ambigu à la ville. Ils oscillent entre pensum énervant par sa répétitivité et valorisation de l'obligation comme traduisant le prix à payer pour leur qualité de vie, sans hésiter d'ailleurs à se déplacer si le besoin se fait sentir, sauf en cas de problèmes financiers spécifiques.

- Le troisième groupe concerne des ménages arrivés plus récemment, généralement assez bien intégrés dans leur commune de résidence, mais ne reniant surtout pas leur origine urbaine. Disposant d'un capital culturel souvent assez important, leurs rapports au village périurbain sont assez intenses, même si l'utilitaire reste le premier facteur de fréquentation de la commune (pour les activités des enfants, etc.), mais ils sont ouverts à des relations sociales dans la commune. Certains ont même une remarquable faculté d'adaptation, pouvant intégrer le conseil municipal dans les trois années qui suivent leur arrivée. Ils défendent souvent une certaine qualité de vie dans leur commune, mais pensent aussi à son développement, notamment en matière d'animation ou même de développement économique. Pourtant, ils se définissent généralement comme urbains, prenant comme référent identitaire le nom de la grande ville. Leur pratique de la ville est intense, n’hésitant pas à revenir le soir pour une sortie ou pour aller chez des amis, alors qu'ils ont travaillé la journée en centre-ville et ont pu faire les courses ou une activité sportive en soirée. Leur appréciation de la mobilité fait que celle-ci n’est pas un obstacle, apparaissant même comme une valeur positive, tant en ce qui concerne la mobilité quotidienne (avec un argumentaire qui tourne souvent autour de la qualité de vie, voire de valeurs environnementales liées à leur lieu de résidence) que pour la mobilité résidentielle. Leur pratique du logement reste pourtant aussi importante que pour les autres groupes, recevant même plus souvent que les autres catégories, mais les personnes invitées viennent autant du reste de la ville que de l'espace environnant. 
- A contrario, le dernier groupe reste vraiment centré sur le logement et uniquement sur celui-ci. Arrivés en même temps que le groupe précédent, c'est-à-dire assez récemment mais souvent plus loin dans le périurbain, ces ménages n’ont ni une pratique très intense de la commune périurbaine où ils habitent, ni une pratique très importante de la ville. La fréquentation de la localité est restreinte à des utilités, avec moins de besoins exprimés que dans le groupe précédent, avec moins de moyens financiers aussi, et les relations sociales avec les voisins sont généralement assez restreintes, souvent limitées à une ou deux familles. Bien qu'ils travaillent aussi très majoritairement en ville (même si beaucoup de conjointes de ce groupe soient assistantes maternelles), celle-ci ne génère que peu de référence identitaire. D’ailleurs, la référence au parcours résidentiel antérieur est plus basée sur des quartiers ou sur une description minutieuse des logements antérieurs que sur la description d'un véritable parcours dans la ville. Les relations sociales sont essentiellement des relations dans le cadre de la famille, malgré parfois un éloignement assez important, d'où l’importance des activités à domicile, télévision, jardinage, bricolage, etc. L'appréciation de la mobilité est généralement plus négative que dans le groupe précédent, surtout si elle devait générer un éloignement de la famille. La mobilité quotidienne est limitée à un seul aller-retour par jour, notamment pour des raisons financières, et la mobilité résidentielle n'est pas envisagée (par exemple en cas de problèmes professionnels) alors même que le hasard a parfois conduit à la localisation résidentielle actuelle. Le logement est donc la référence identitaire principale et l'autodéfinition de l'identité spatiale est peu convaincante, cherchant parfois l'assentiment de l'interlocuteur et se basant essentiellement sur les territoires administratifs avec en particulier le département.

\section{Conclusion}

Le bilan sur l'identité sociale et spatiale des habitants du périurbain est donc contrasté, loin des discours généraux qui feraient de cet espace une entité parfaitement homogène. Au sein de l'espace périurbain, il existe de multiples plans de différenciation. Le premier fonctionne à partir de la composition sociale, qui varie essentiellement selon la distance au centre, avec une décroissance régulière des catégories moyennes et une augmentation régulière des ouvriers, le marché immobilier contribuant à la constitution d'une couronne de catégories populaires solvables. Le second axe de différenciation se forme selon les segments du marché immobilier, avec une relation forte entre type d'habitat et groupes sociaux, ce qui rapproche le mode de fonctionnement du marché immobilier périurbain de celui du marché immobilier urbain. Le troisième facteur de différenciation est basé sur la façon d'habiter le périurbain, avec des rapports toujours forts au logement, caractéristiques d'une population avec de fortes proportions de familles avec enfants, mais les pratiques du village périurbain et de la ville sont très différenciées, allant de l'absence totale d'intégration à l'implication continue. Enfin, il existe des identifications spatiales variées, entre rural et urbain, notamment selon l'ancienneté de l'installation dans le périurbain et la nature du parcours antérieur. L'articulation entre identité urbaine et identité rurale n'est donc jamais univoque, elle peut prendre la forme de l'émergence d'un véritable habiter périurbain en particulier pour les ménages les plus anciennement installés dans ce type d'espace, mais elle peut prendre aussi la forme d'une simple déclinaison de pratiques existantes, qu'elles soient spécifiquement rurales pour les ménages qui ne sont pas ou peu passés par la ville dans leur parcours résidentiel antérieur, ou qu'elles soient spécifiquement urbaines pour des ménages qui considèrent le périurbain comme une simple alternative, souvent liée à un moment spécifique du cycle de vie du ménage, à des localisations urbaines centrales ou périphériques.

\section{Bibliographie}

Authier (J.-Y.) (dir.), 2001. - Du domicile à la ville, vivre en quartier ancien, Anthropos, 209 p. 
RODOLPHE DODIER

Arlaud (S.), Jean (Y.), Royoux (D.) (dir.), 2005. - Rural-Urbain, nouveaux liens, nouvelles frontières, Rennes, PUR, $506 \mathrm{p}$.

Baudelle (G.), Darris (G.), Ollivro (J.), Pihan (J.), 2004. - « Les conséquences d'un choix résidentiel périurbain sur la mobilité : pratiques et représentations des ménages », Cybergéo, n 287, $17 \mathrm{p}$.

Berger (M.), 1991. - «L'urbanité des périurbains d'Ile-de-France : de la diversité à la ségrégation », Les Annales de la Recherche Urbaine, $\mathrm{n}^{\circ}$ 50, p. 56-63.

Berger (M.), 2004. - Les périurbains de Paris, Paris, CNRS, 317 p.

Bertrand (J.-R.), Chevalier (J.), Dodier (R.), Gasnier (A.), 2000. - Le Mans. Peut-on changer la ville?, Anthropos, $220 \mathrm{p}$.

Bonvalet (C.), Dureau (F.), 2000. - « Les modes d'habiter : des choix sous contraintes », dans Dureau (F.) et al. (dir.), Métropoles en mowvement, Paris, Anthropos, p. 131-153.

Di Méo (G.), 1998. - Géographie sociale et territoires, Nathan, 317 p.

Dodier (R.), 2004a. - «L'espace dans les stratégies résidentielles des ménages », ESO Travaux et documents, $n^{\circ} 21$, p. 115-123.

Dodier (R.), 2004b. - « Migrations des cadres, entre métropolisation et diffusion », Bulletin de l'Association de Géographes Français - Géographies, n² 2, p. 182-192.

Dodier (R.), 2005. - « Mobilité quotidienne de travail : du couple ville industrielle - espace rural à la « nouvelle » organisation urbaine », dans ARLaud (S.), Jean (Y.), Royoux (D.) (dir.), Rural - Urbain. Nouveaux liens, nouvelles frontières, Rennes, PUR, p. 71-82.

ESTÈBE (P.), 2004. - «Quel avenir pour les périphéries urbaines? », Esprit, n 303, p. 82-95.

Frémont (A.), 1976. - La région, espace vécu, Paris, PUF, 2e édition 1998, 288 p.

Guilluy (C.), Noyé (C.), 2004. - Atlas des nowvelles fractures sociales en France, Paris, Autrement, 64 p.

Haegel (F.), 1998. - « Une lecture spatiale des identités », dans Haumont (A.) (dir.), L'urbain dans tous ses états. Faire, vivre, dire la ville, Paris, L'Harmattan, p. 203-220.

Haumont (N.), 2001. - Les pavillonnaires : étude psychologique d'un mode d'habitat, Paris, L'Harmattan, édition originale, 1966, CRU, 150 p.

Hoyaux (A.-F.), 2002. - « Entre construction territoriale et constitution ontologique de l'habitant : introduction épistémologique aux apports de la phénoménologie au concept d'habiter », Cybergéo, n 102, 18 p.

JAiLlet (M.-C.), 2004. - «L'espace périurbain : un univers pour les classes moyennes », Esprit, n 303, p. $40-60$.

LÉvy (J.), Lussault (M.) (dir.), 2003. - Dictionnaire de la géographie et de l'espace des sociétés, Paris, Belin, 1034 p.

Moles (A.), 1992. - «Vers une psycho-géographie », dans Bailly (A.), Ferras (R.), Pumain (D.) (dir.), Encyclopédie de géographie, Paris, Economica, p. 177-205.

Moles (A.), Rohmer (E.), 1998. - Psychologies de l'espace, Paris, L'Harmattan, 158 p.

MoreAu-LANÉELLE (X.), 2003. - Les migrations alternantes : stratégies et contraintes économiques et sociales, Thèse de doctorat, EHESS, 453 p.

Pinson (D.), Thomann (S.), 2002. - La maison en ses territoires, de la villa à la ville diffuse, Paris, L'Harmattan, $191 \mathrm{p}$.

Raymond (H.), 1998. - « Habiter et vie quotidienne », dans Logement et habitat, l'état des savoirs, Paris, La Découverte, p. 390-396.

RAYMOND (S.), 2003. - Du retour à la nature au retour à la campagne, migrants et recompositions territoriales dans le Midi de la France et en Californie du Nord, Thèse de doctorat, Université de Toulouse Le Mirail, 352 p.

Rougé (L.), 2005. - « Les nouvelles frontières du périurbain : accession à la propriété en maison individuelle et pratiques résidentielles des ménages toulousains », dans ArLaud (S.), JEAN (Y.), Royoux (D.) (dir.), Rural - Urbain. Nowveaux liens, nowvelles frontières, Rennes, PUR, p. 137-145.

Segaud (M.), Brun (J.), Driant (J.-C.) (dir.), 2003. - Dictionnaire de l'habitat et du logement, Paris, Colin, $451 \mathrm{p}$.

Cet article a été reçu le 30 juin 2006 et définitivement accepté le 15 janvier 2007. 